\title{
Indirect techniques for astrophysical reaction rates determinations
}

F. Hammache ${ }^{1}$, N. Oulebsir ${ }^{1,2}$, S. Benamara ${ }^{3,1}$, N. De SÉréville ${ }^{1}$,

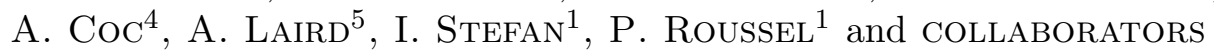

1 IPN, IN2P3-CNRS, Université Paris sud 11, 91406 Orsay, France

${ }^{2}$ LPT, Université A. Mira, 06000 Béjaia, Algérie

${ }^{3}$ LPCQ, Université Mouloud Mammeri, Tizi-Ouzou, Algérie

${ }^{4}$ CSNSM, IN2P3-CNRS, Université Paris Sud 11, 91405 Orsay, France

${ }^{5}$ Department of Physics, University of York, York, UK

\begin{abstract}
Direct measurements of nuclear reactions of astrophysical interest can be challenging. Alternative experimental techniques such as transfer reactions and inelastic scattering reactions offer the possibility to study these reactions by using stable beams. In this context, I will present recent results that were obtained in Orsay using indirect techniques. The examples will concern various astrophysical sites, from the Big-Bang nucleosynthesis to the production of radioisotopes in massive stars.
\end{abstract}

The main characteristics of the nuclear reactions involved in stellar nucleosynthesis is the low energy where they generally occur, between few $\mathrm{keV}$ to few $\mathrm{MeV}$ and at these energies the cross sections of these reactions are very small ranging from fbarn to hundreds of pbarn especially when it involves charged particles. This makes the direct measurements at stellar energies very difficult and often impossible. Hence direct measurement are usually performed at higher energies and then extrapolated down to stellar energies where the reactions occur. However the extrapolations can lead sometimes to wrong results when a possible very low energy resonance or the tail of a possible sub-threshold resonance are not taken into account. 
The effect of these resonances may change the extrapolated S-factor by a huge factor. The other problem with direct measurements is related to the radioactive nature of the nuclei involved in reactions occurring for instance in novae, $\mathrm{r}$-process,... The radioactive beam intensities are usually low, they rarely exceed $10^{5}-10^{6}$ pps and for nuclei which have relatively long half lives, making targets with enough at $/ \mathrm{cm}^{2}$ is quite difficult.

To bypass all these difficulties, related to extrapolation and/or radioactive nuclei, indirect techniques such as transfer reactions, ANC, inelastic scattering and charge exchange-reactions may help to determine the most important ingredients to evaluate the cross section and reaction rates of resonant reactions: the energy level, spin-parity and partial decay widths of the state of interest in the formed compound nucleus.

In the following, three cases of astrophysical interest that were studied in Orsay using indirect techniques will be presented.

\section{Study of ${ }^{12} \mathbf{C}(\alpha, \gamma){ }^{16} \mathrm{O}$ through $\alpha$-transfer reaction}

The importance of the challenging ${ }^{12} \mathrm{C}(\alpha, \gamma){ }^{16} \mathrm{O}$ reaction in energetics, nucleosynthesis and final fate of the massive stars is notorious [1]. Despite the various experiments [2] which studied this reaction, its cross section remains highly uncertain. At the Gamow peak of $300 \mathrm{keV}$ where the reaction occurs during the He burning stage, the expected cross section is about $10^{-7}$ nbarn which means impossible to measure directly. Hence direct measurements were performed down to $900 \mathrm{keV}$ in center of mass energy and then extrapolated down to $300 \mathrm{keV}$ using R-matrix formalism. But the extrapolation in this case is very complicated. Indeed, the capture cross section at $300 \mathrm{keV}$ which correspond to the excitation energy of $7.46 \mathrm{MeV}$ in ${ }^{16} \mathrm{O}$ is dominated by the overlap of several contributions, the most important ones being the E1 and E2 transitions to the ground state via the low energy tail of the $1^{-}$broad state at $9.6 \mathrm{MeV}$ of ${ }^{16} \mathrm{O}$ and the high energy tails of the $1^{-}$and $2^{+}$sub-threshold states at 6.92 and $7.12 \mathrm{MeV}$ of ${ }^{16} \mathrm{O}$. The effect of these two states was badly known because their alpha-spectroscopic factors and so their alpha-reduced widths were spread over a large range of values.

In view of the large uncertainties surrounding the alpha-spectroscopic factors of the two sub-threshold states, a new measurement of these parameters was performed through the $\alpha$ transfer reaction $\left.{ }^{12} \mathrm{C}\left({ }^{7} \mathrm{Li}, \mathrm{t}\right)\right)^{16} \mathrm{O}[3]$.

The experiment [3] was performed at the Alto facility of Orsay using the magnetic Split-Pole spectrometer. A ${ }^{7} \mathrm{Li}$ beam was sent on a self-supporting enriched ${ }^{12} \mathrm{C}$ target. The reaction products were momentum analyzed by 
the Split-Pole and the tritons were detected in the focal plane first by a position sensitive gas chamber and then by a $\Delta \mathrm{E}$ proportional counter. The angular distribution measurements were performed at two incident energies, 34 and $28 \mathrm{MeV}$, and at angles up to $45^{\circ}$ in $\mathrm{cm}$ in order to check the direct character of the transfer mechanism.

In Fig. 1 are displayed the measured differential cross sections for 4 populated states of ${ }^{16} \mathrm{O}$ at the two incident energies together with the FRDWBA calculations performed with FRESCO code in dotted curves and HF calculations in dotted-dashed curves. The solid curves are the incoherent sum of the two contributions.

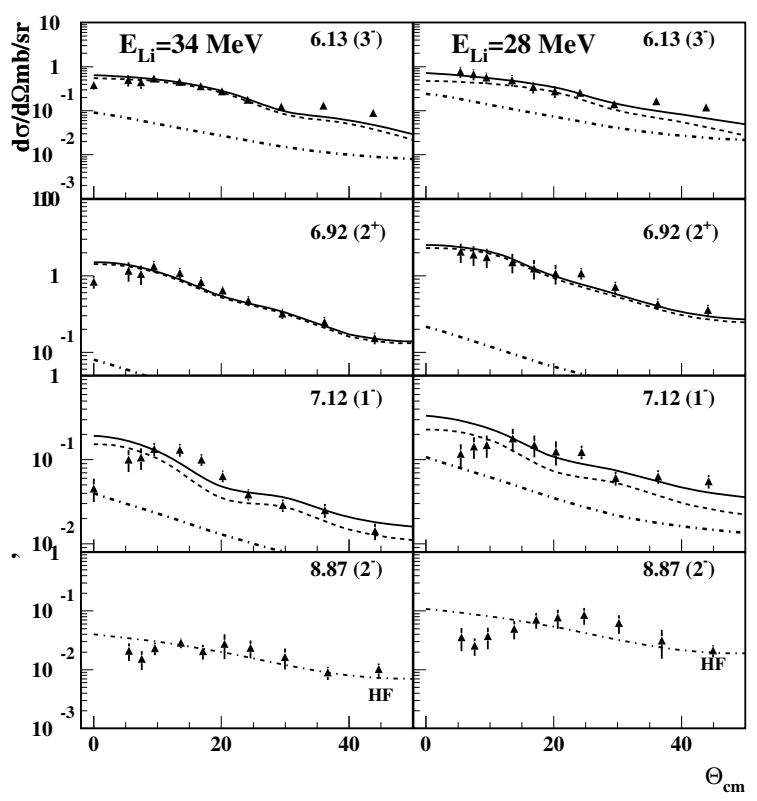

Figure 1: Experimental differential cross sections of the ${ }^{12} \mathrm{C}\left({ }^{7} \mathrm{Li}, \mathrm{t}\right){ }^{16} \mathrm{O}$ reaction obtained at $34 \mathrm{MeV}$ (left column) and $28 \mathrm{MeV}$ (right column) for the 6.13, 6.92, 7.12 and $8.87 \mathrm{MeV}$ states of ${ }^{16} \mathrm{O}[3]$.

One can see, that except for the non-natural parity state at $8.87 \mathrm{MeV}$, the data are quite well described by the FR-DWBA calculation at the two incident energies and this indicates that the direct transfer mechanism is the dominant one in this transfer measurement.

The $\mathrm{S}_{\alpha}$ of the two sub-threshold states were extracted from the normalization of the FR-DWBA calculations to the data. The values obtained for 
the $6.92 \mathrm{MeV}$ and $7.12 \mathrm{MeV}$ states, $27 \pm 10 \mathrm{keV}$ and $8 \pm 3 \mathrm{keV}$ respectively, were then used to calculate their $\alpha$-reduced widths at the radius of $6.5 \mathrm{fm}$ where the radial part of the $\alpha^{-12} \mathrm{C}$ cluster wave function reaches its asymptotic behavior. The assymptotic normalization coefficients (ANC) of the two states of interest were also deduced, $\mathrm{C}^{2}(6.92 \mathrm{MeV})=(2.07 \pm 0.80) \times 10^{10}$ $\mathrm{fm}^{-1}$ and $\mathrm{C}^{2}(7.12 \mathrm{MeV})=(4.00 \pm 1.38) \times 10^{28} \mathrm{fm}^{-1}$ and were found to be in agreement with the results of ANC experiments of Brune et al [4] and the most recent ones of Avila et al [5].

The obtained $\alpha$-reduced widths of the $2^{+}$and $1^{-}$sub-threshold states were included in an R-matrix calculation of the E1 and E2 component of ${ }^{12} \mathrm{C}(\alpha, \gamma){ }^{16} \mathrm{O}$ using Descouvemont code. The details of the R-matrix calculations and the fitting procedure of the E2 and E1 measured phase shifts and astrophysical S-factors is given in [3]. The deduced S-factors at 300 $\mathrm{keV}$ for the $\mathrm{E} 2$ and $\mathrm{E} 1$ components, $50 \pm 19 \mathrm{keV}-\mathrm{b}$ and $100 \pm 28 \mathrm{keV}$ respectively, were found in excellent agreement with those deduced in the ANC experiment of Brune et al. [4] where the alpha-reduced widths of the two sub-threshold states were also fixed in the R-matrix fitting procedure. The obtained values are also in agreement within the error bars with the values recommended in the very recent updated Nacre compilation (NACRE II) $[6] ; \mathrm{S}_{E 2}(300 \mathrm{keV})=61 \pm 19 \mathrm{keV}-\mathrm{b}$ and $\mathrm{S}_{E 1}(300 \mathrm{keV})=80 \pm 18 \mathrm{keV}-\mathrm{b}$.

\section{The ${ }^{7} \mathrm{Li}$ cosmological problem}

The primordial nucleosynthesis of light elements, D, ${ }^{3,4} \mathrm{He}$ and ${ }^{7} \mathrm{Li}$, is one of the three observational pillars of the Big-Bang model. The observed primordial abundances for D and ${ }^{3,4} \mathrm{He}$ agree well with the predictions of BBN model together with the precise WMAP cosmic baryon density while ${ }^{7} \mathrm{Li}$ abundance observations in metal poor halo stars are three times smaller than BBN+WMAP predictions [7]. This discrepancy is what is known as the ${ }^{7} \mathrm{Li}$ problem. Many ideas and explanations were addressed to explain the ${ }^{7} \mathrm{Li}$ deficit but none of them were satisfactory.

${ }^{7} \mathrm{Li}$ is produced mainly by ${ }^{7} \mathrm{Be} \mathrm{EC}$ decay and the most important reactions which produces and destroys ${ }^{7} \mathrm{Be}$ are ${ }^{3} \mathrm{He}\left({ }^{4} \mathrm{He}, \gamma\right){ }^{7} \mathrm{Be}$ and ${ }^{7} \mathrm{Be}(\mathrm{n}, \mathrm{p}){ }^{7} \mathrm{Li}(\mathrm{p}, \alpha) \alpha$ respectively. The cross section of ${ }^{3} \mathrm{He}\left({ }^{4} \mathrm{He}, \gamma\right){ }^{7} \mathrm{Be}$ reaction was measured by several groups using different techniques and its knowledge is nowadays better than $8 \%$ [8]. The cross sections of ${ }^{7} \mathrm{Be}(\mathrm{p}, \mathrm{n})^{7} \mathrm{Li}$ and ${ }^{7} \operatorname{Li}(\mathrm{p}, \alpha) \alpha$ reactions are also well known [9]. Recently some authors [10-13] proposed the idea that some missed resonances in secondary destruction channels of ${ }^{7} \mathrm{Li}$ and ${ }^{7} \mathrm{Be}$ could be at the origin of the ${ }^{7} \mathrm{Li}$ deficit. Three 
promising candidates came out from their investigation: ${ }^{7} \mathrm{Be}+\mathrm{d},{ }^{7} \mathrm{Be}+{ }^{3} \mathrm{He}$ and ${ }^{7} \mathrm{Be}+{ }^{4} \mathrm{He}$. The ${ }^{7} \mathrm{Be}+\mathrm{d}$ reaction channel was investigated by various works ( [14] and references therein) and it was dismissed. Hence we took care about the two other candidates in Orsay for which a presence of a narrow $1^{-}$or $2^{-}$state close to $15 \mathrm{MeV}$ in the compound nucleus ${ }^{10} \mathrm{C}$ or a presence of a state close to $7.8 \mathrm{MeV}$ in the compound nucleus ${ }^{11} \mathrm{C}$ may reconcile the ${ }^{7} \mathrm{Li}$ predictions with the observations.

The search for the missing resonant states has been performed at the split-pole (SP) spectrometer of the ALTO facility. ${ }^{10} \mathrm{C}$ and ${ }^{11} \mathrm{C}$ nuclei were populated with the $\left({ }^{3} \mathrm{He}, \mathrm{t}\right)$ charge exchange reaction on ${ }^{10} \mathrm{~B}$ and natural Boron targets at $35 \mathrm{MeV}$ and the emitted tritons were detected at four SP angles [15].

In Fig. 2 and Fig. 3 are displayed the measured $\mathrm{B} \rho$ position spectra for ${ }^{11} \mathrm{C}$ and ${ }^{10} \mathrm{C}$. The well populated peaks observed in ${ }^{11} \mathrm{C}$ spectrum are the already well known ${ }^{11} \mathrm{C}$ levels and no new peak is seen in the energy region of interest between 7.79 and $7.9 \mathrm{MeV}$. Thanks to the very large signal to background ratio observed in the spectrum, one can assert that it is very unlikely that a new state in ${ }^{11} \mathrm{C}$ exists in the energy region of investigation. Moreover, all known ${ }^{11} \mathrm{~B}$ states below $\mathrm{Ex}=9 \mathrm{MeV}$ have their counterpart in ${ }^{11} \mathrm{C}$ mirror nucleus.

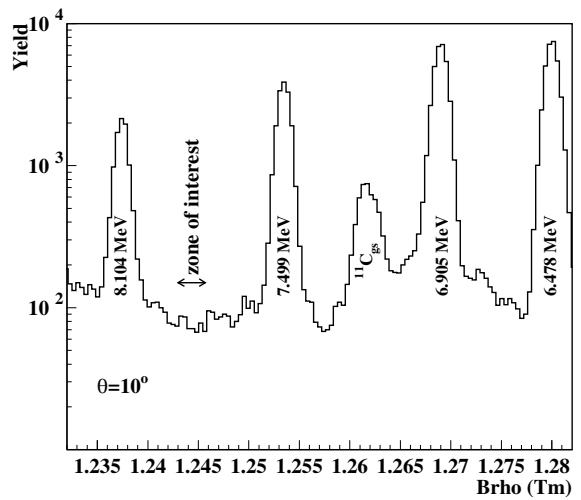

Figure $2:{ }^{11} \mathrm{C} \mathrm{B} \rho$ position spectrum measured at $10^{\circ}$.

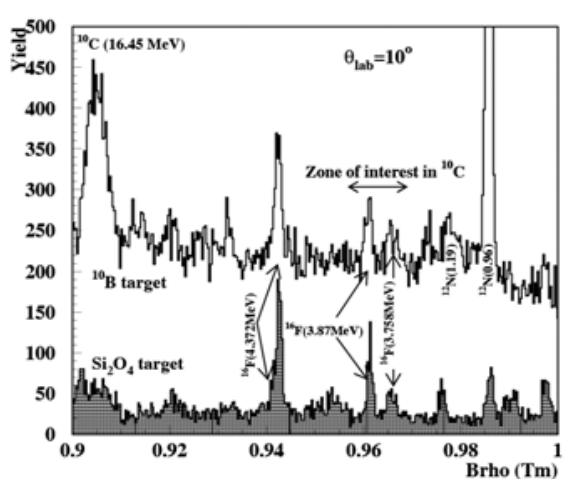

Figure 3: $\mathrm{B} \rho$ position spectra measured at $10^{\circ}$ on ${ }^{10} \mathrm{~B}$ and $\mathrm{Si}_{2} \mathrm{O}_{4}$ targets.

No additionnal states in ${ }^{10} \mathrm{C}$ are observed in Fig. 3 around $15 \mathrm{MeV}$ neither, the only states we observe are those of ${ }^{16} \mathrm{~F}$ populated via $\left({ }^{3} \mathrm{He}, \mathrm{t}\right)$ on the ${ }^{16} \mathrm{O}$ contamination of the target as one can see from the grey filled histogram obtained with $\mathrm{Si}_{2} \mathrm{O}_{4}$ target. The same results were obtained at the other measured angles. But since the background in this case is 
very important and the signal to background ratio is 10 times less than in the case of ${ }^{11} \mathrm{C}$, one can not exclude that a state may be hidden in the background. However, from a $\chi^{2}$ study [15] of a simulated state close to $15 \mathrm{MeV}$ with various widths and populated cross sections on the top of the measured background, we could conclude that any $1^{-}$or $2^{-}$state of ${ }^{10} \mathrm{C}$ in the excitation energy region of interest has very likely, if present, a total width larger than $590 \mathrm{keV}$ to escape our detection. With this limit and even a three times lower one, the calculated ${ }^{7} \mathrm{Be}+{ }^{3} \mathrm{He}$ reaction rates [15] do not solve the ${ }^{7} \mathrm{Li}$ problem. Finally, from our results we conclude that the ${ }^{7} \mathrm{Li}$ problem is not due to resonant states in ${ }^{10} \mathrm{C}$ and ${ }^{11} \mathrm{C}$. With this conclusion and those of previous works concerning other reactions, we may even say that the solution has very likely to be found outside of nuclear physics.

\section{$3 \quad{ }^{26} \mathrm{Al}$ nucleosynthesis in massive stars}

Massive stars are large producers of the ${ }^{26} \mathrm{Al}$ radioisotope which is a $\beta^{+}$emitter decaying to ${ }^{26} \mathrm{Mg}$ g.s. after emitting a characteristic $\gamma$-ray line at 1.809 $\mathrm{MeV} .{ }^{26} \mathrm{Al}$ has been observed since the late $70 \mathrm{~s}$ in the galactic plane and its emission has been correlated to massive star population. It is also observed through excesses of its daughter nucleus ${ }^{26} \mathrm{Mg}$ in meteorites inclusions.

The interpretation of both these observations need precise yields of ${ }^{26} \mathrm{Al}$. In massive stars, ${ }^{26} \mathrm{Al}$ is mostly produced during explosive burning and it was shown that the ${ }^{26} \mathrm{Al}$ yield depends crucially on the ${ }^{26} \mathrm{Al}(\mathrm{n}, \mathrm{p})$ and ${ }^{26} \mathrm{Al}(\mathrm{n}, \alpha)$ reactions rates [16]. Unfortunately, the latters are badly known because of the lack of spectroscopic information in the ${ }^{27} \mathrm{Al}$ compound nucleus $(\mathrm{CN})$ above the neutron threshold.

For typical temperatures of 2 billion degrees achieved during explosive burning, resonances of interest in the $\mathrm{CN}{ }^{27} \mathrm{Al}$ are located from neutron threshold (at $13 \mathrm{MeV}$ ) to about $500 \mathrm{keV}$ above neutron threshold. Several direct and time reverse measurements of these reactions were performed but the covered energy range did not extend up to $500 \mathrm{keV}$ above neutron threshold in the center of mass. Moreover, the experimental ${ }^{26} \mathrm{Al}(\mathrm{n}, \mathrm{p})$ reaction rates show a disagreement between the different sets of measured data [17]. Hence an improvement of the knowledge of ${ }^{27} \mathrm{Al}$ spectroscopy is really needed.

The excited states of ${ }^{27} \mathrm{Al}$ were populated at the Alto facility of Orsay via ${ }^{27} \mathrm{Al}(\mathrm{p}, \mathrm{p}$ ') reaction [18] at $18 \mathrm{MeV}$ incident energy and using Split-Pole spectrometer for the detection of the protons. In total, more than 30 new levels were observed above the neutron threshold (see Fig.4 and Fig.5 in ref 
[18]). The Orsay Split-Pole results were confirmed by the recent ${ }^{27} \mathrm{Al}(\mathrm{p}, \mathrm{p}$ ') measurements performed at the Q3D of MLL Munich where a better energy resolution $(7 \mathrm{keV})$ and a better energy precision $(2 \mathrm{keV})$ could be achieved. New states were observed in the Q3D experiment due to the improvement in the energy resolution as one can see in Fig. 4 where a small part of the measured spectra with the Split-Pole and the Q3D spectrometers are displayed.
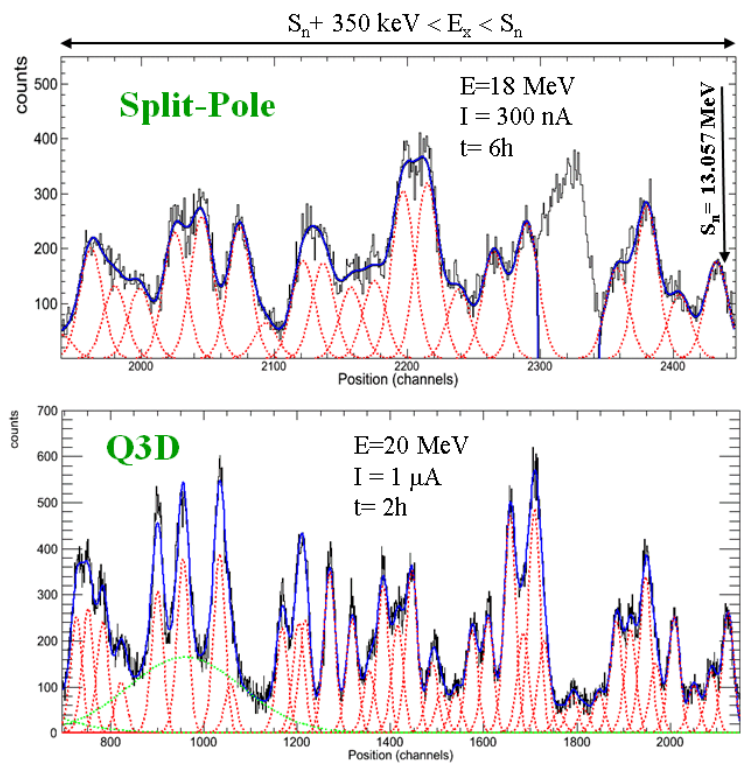

Figure 4: $\mathrm{B} \rho$ position spectra of ${ }^{27} \mathrm{Al}$ measured with Split-Pole in Orsay (top) and with Q3D in MLL Munich (bottom). The dotted curves are fit of the spectra after background substraction.

A coincidence measurement coupling the SP spectrometer with three DSSSDs placed around the target in a close geometry was performed recently in order to study the decay modes of the measured new resonances and try to extract the decay branching ratios needed for reaction rates calculations. The analysis of the data is still in progress.

\section{Acknowledgments}

I would like to thank all my collaborators from IPNO, CSNSM, York, Ganil, ASCR, Algeria,Barcelona, Huelva, Santiago de Compostella and Saha Insti- 
tut who participate in the experiments described in this contribution.

\section{References}

[1] T.A. Weaver and S.E. Woosley, Phys. Reports 227 (1993) 65.

[2] A. Belhout et al., Nucl. Phys. A793 (2007) 178 and references therein.

[3] N. Oulebsir, F. Hammache et al., Phys. Rev. C 85 (2012) 035804.

[4] C. R. Brune, W. H. Geist, R. W. Kavanagh and K. D. Veal, Phys. Rev. Lett. 83 (1999) 4025.

[5] M.L.Avila, G.V.Rogachev et al., Phys.Rev.Lett. 114 (2015) 071101.

[6] Y. Xu, K. Takahashi, S.Goriely et al., Nucl. Phys. A 918 (2013) 61

[7] A. Coc, E. Vangioni, J. Cosmology. Astropart. Phys. 10 (2014) 050.

[8] E. G. Adelberger et al., Rev. Mod. Phys. 83 (2011) 195.

[9] P. Descouvemont, A. Adahchour, C. Angulo, A. Coc and E. VangioniFlam, At. Data Nucl. Data Tables 88 (2004) 203.

[10] N. Chakraborty, B. D. Fields, K. Olive, Phys. Rev. D 83 (2011) 063006.

[11] C. Broggini, L. Canton, G. Fiorentini, F.L. Villante, J. Cosmology Astropart. Phys. 06 (2012) 030.

[12] O. Civitarese, M.E. Mosquera, Nucl. Phys. A 898 (2013) 1

[13] R.H. Cyburt, M. Pospelov, Int. J. Mod. Phys. E 21 (2012) 1250004.

[14] O. S. Kirsebom, B. Davids, Phys. Rev. C 84 (2011) 058801 and referrences therein.

[15] F. Hammache, A. Coc et al., Phys. Rev. C 88 (2013) 062802(R).

[16] C. Iliadis, A. Champagne, A. Chieffi, and M. Limongi, Astrophys. J. Suppl. 193 (2011) 16.

[17] B. M. Oginni, C. Iliadis et al. Phys.Rev.C 83,025802 (2011)

[18] S. Benamara, N.de Sereville, et al., Phys.Rev. C 89 (2014) 065805. 